\title{
Extracting Dynamic Microtubule Features from Image Sequences
}

\author{
Alphan Altınok \\ M. Emre Sargin \\ B. S. Manjunath \\ Kenneth Rose \\ Electrical and Computer Engineering, University of California Santa Barbara, Santa Barbara, CA, 93106 \\ \{alphan, msargin, manj, rose\}@ece.ucsb.edu
}

Funded by the Center for Bioimage Informatics under NSF-ITR 0331697.

\begin{abstract}
Microtubules (MT) are filamentous cytoskeletal structures composed of tubulin protein subunits. Through their dynamic behaviors, they participate in many essential cellular functions. MT dynamics are traditionally analyzed from time lapse images by manual techniques that are laborious, approximate and often of limited analytical value. Recently, computational methods have been applied to the problems of detection and tracking of MTs in live cell images. In this paper, we discuss computational methods for extracting dynamics features from live cell MT image sequences. We provide examples of how these features can be used in MT dynamics analysis and improve the manual analytical capabilities.
\end{abstract}

\section{Introduction}

Fluorescence imaging is a mainstream data acquisition tool in cell biology. This high throughput data channel produces an immense amount of data every year. However, the analysis of the biological phenomena remains largely a manual task. Quantitative studies are often subject to variability, user bias, approximation errors, and limited manual measurement and analysis capabilities, while requiring substantial time and effort to achieve statistical significance. Thus, computer vision based tools find pertinent applications in the automated analysis of biological image collections. Furthermore, biological experimentation becomes more and more centered around the newly available computational methods. Here, we discuss recent methods proposed for the data collection and analysis aspects of microtubule (MT) image sequences.

As a major component of the cytoskeleton, MTs are tubular polymers composed of $\alpha$ and $\beta$ tubulin dimers associated by non-covalent interactions. They measure $25 \mathrm{~nm}$ in diameter and several $\mu m$ to $m m$ in length. MTs participate in many critical cellular functions through changing their length, Fig. 1. The alternation between the growing and shortening cycles is commonly referred as the MT dynamic instability. These dynamic behaviors are finely regulated within the cell by the MT-associated proteins. For example, recognition of the tubulin conformation at the MT tip is a model mechanism by which the tip-tracking proteins are predicted to regulate MT dynamics. MT-targeted drugs are pharmacological agents developed with the objective of regulatory operation.

Research on MT dynamics seeks to understand underlying cellular mechanisms relating to normal and abnormal functioning of the cell in response to changes in environmental conditions [5] through the regulatory changes instigated by the numerous agents. A common conjecture is that certain vital diseases such as Alzheimer's and cancer are at least correlated with the regulatory abnormalities in the MT dynamics. For further structural and functional details we refer the reader to [2].

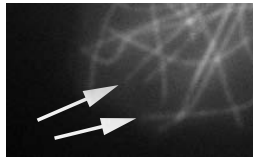

(a)

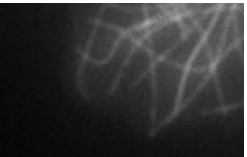

(b)

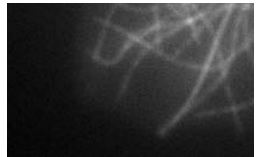

(c)
Figure 1. Consecutive frames from a MT video. The marked MTs change their length in subsequent frames.

MT dynamic behaviors are quantified by collecting growth and shortening statistics of MT populations from time-lapse image sequences. The primary technique is to manually track the tip positions of individual MTs over the image sequence. Then, the tip positions are converted to MT length estimates as the Euclidean distance from a point on the MT body that is fixed throughout the video. These estimates are graphed as statistics of recording time per MT, termed as the life history plot. The statistics, including the rates and extents of dynamic events and the frequencies of switching between the types of events are computed for MT populations from the life history plots. This aggregated information is used in determining the ability of the treatment agent to promote or suppress MT dynamics. For example, paclitaxel (Taxol) -a cancer therapeutic- is shown to stabi- 
lize MT dynamics, [7, 8].

While the immediate goal is to assist the user with the analysis, it may be possible to boost the analysis efficacy beyond the manual practice. Specifically, it is possible to quantify data at a richer level than what is manually feasible. For example, once the MT body is segmented curvature variations can be computed easily, where such features are not feasible to calculate manually. In the rest of this paper we describe the MT tracking and time series analysis problems and related challenges. We report on recent approaches addressing these problems.

\section{Methods}

Biological content presents specific challenges. For example, despite highly controlled image acquisition, image quality is variable. In live cell imaging, the experimenter has little control on the spatial distribution of objects. Furthermore, typical images consist of $2 \mathrm{D}$ projections of $3 \mathrm{D}$ biological structures, resulting in a mesh of image objects where intersections, occlusions, and similar visual challenges become a norm rather than exception.

Many curve or contour detection techniques remain ineffective in segmenting the MT body due to a combination of excessive natural clutter and frequent local intensity variations. In this section, we discuss two tracking methods: [3] uses active contours for extracting the MT length as open ended curves, and [10] uses a hidden Markov model based approach for tracking the deformation of a curve after initially tracing it on the first frame. From the usage point of view, the two methods provide different user interaction stipulations. The first technique is less amenable for user interaction than the second one.

\subsection{Automated tracking}

We define the MT tracking problem as following the movements of an individual MT body over the frames of a video. The goal is to quantify the changing patterns of MT lengths during the observation period. Thus, lengths must be measured in each frame. Manual practice is to estimate the length as the Euclidean distance between the tip position and an arbitrary origin that is fixed throughout the frames. In computational domain the length can be measured as the arc length along the MT body, Fig. 5. Note that the problem of tracking MT tips is different than tracking MT bodies.

In [3], the tracking method consists of the following components:

- detecting the MT tips,

- matching tip positions on different frames,

- extracting MT body from tip tracks.

The rationale behind this approach is that MT tip detection has a better success rate than that of MT segmentation over the entire video. Note that this may not be true for any given frame. Once the tips are located and matched then the body extraction problem is simplified since only those MTs that were found to have consistent tip tracks are to be extracted.

The tip detection problem is addressed as follows. MT body is assumed to be a locally linear structure. Thus, a line filter, second derivative of Gaussian, is applied to the image for enhancing the polymer mass of the MT network. If the intensity of a pixel at $(x, y)$ is denoted by $I(x, y)$, then the filter response is given by

$$
I^{f}(x, y)=\max _{\theta}\left(I(x, y) * G_{\sigma, \theta}^{\prime \prime}(x, y)\right)
$$

where $G_{\sigma, \theta}^{\prime \prime}(x, y)$ is the second derivative of the Gaussian kernel with scale $\sigma$ and the derivative taken along an orientation $\theta$ at position $(x, y)$. From the binarized and thinned response image all candidate tip positions are recorded. The candidates are then further classified for their potential to be actual tips. Example results from consecutive frames are shown in Fig. 2.

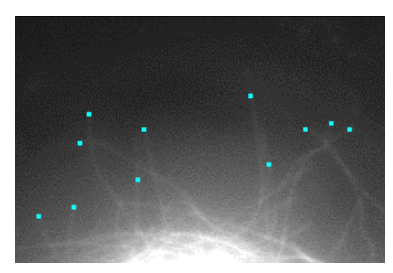

(a)

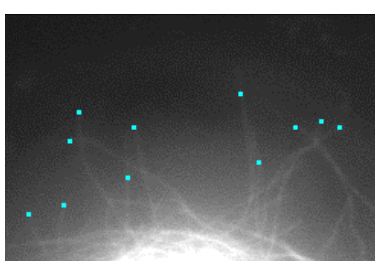

(b)
Figure 2. Tip detection results in consecutive frames from a MT video.

Once the tip positions are located on all video frames, the next step is to find correspondences between the tip positions to establish tip tracks. The crucial problem is to be able to find a method that is flexible enough to handle occasional false positives or true negatives of the tip detection. This is handled by a multi-frame graph matching approach. A graph $G=(V, E)$ is constructed with the vertices $V$ as tip positions in all frames and the edges $E$ as the similarities computed from positional distances between vertices. The edge weights of the graph represent the matching potential of corresponding two tips in different frames. Note that the frames do not need to be consecutive for estimating edge weights. Thus, MT tip tracks can be generated with missing frames. Each tip can be assigned to at most one final tip track. A vertex disjoint path cover $\mathcal{C}$ is a covering of $G$ where each vertex of $G$ is in one and only one path of $\mathcal{C}$. The weight of a path cover is defined as the sum of weights of its edges. Thus, finding the best MT tip tracks corresponds to finding the maximum weight path cover of $G$.

Given the tip track of a MT over the frames of a video, 
its length is estimated by extracting MT body as follows. Let $T_{t}$ denote the position of the tip $T$ in frame $t$, and $O$ denote an arbitrary origin on the MT body that is fixed over the frames. We define the MT body as the curves $C_{t}\left(O, T_{t}\right)$ between $O$ and $T_{t}$. The body extraction refers to finding a suitable $O$ and adjusting $C_{t}$ over the frames $t$. $O$ is estimated on the initial frame by maximizing a geodesic distance function from $T_{1}$ within a window of interest via Fast Marching, [11]. Then, the $C_{t}$ are estimated by using an open-ended active contour model. Using Fast Marching on intensity may lead to erroneous traces, Fig. 3. Note that the $C_{t}$ are not required to correspond to the actual MT body for the purpose of determining the MT growth or shortening from the differences in length.

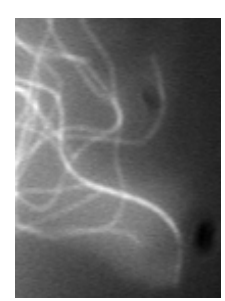

(a)

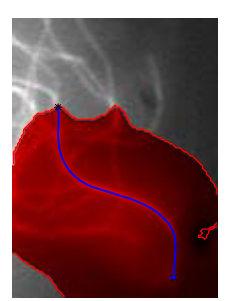

(b)

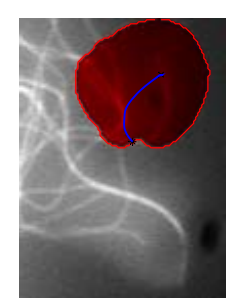

(c)
Figure 3. Example results of Fast Marching. (a) shows the original region. Fast Marching was run on the marked MT segment between two points. The resulting search space is shown in (b). with the shortest path marked between the points. Similar result on another MT is shown in (c), where the shortest path is swayed by the bright spots on a neighboring MT.

If a tip was missed in some frame of the video, then the MT length cannot be computed reliably for that frame. Occasional missing data points should not have a significant effect on the time series analysis.

\subsubsection{Evaluation of automated tracking}

Visual tracking results are shown in Fig. 4. A quantitative evaluation of this method on 26 MTs against manually tracked data revealed a mean error rate of 2.85 pixels with a standard deviation of 4.36. This error level is acceptable since the images capture the fluorescence emissions from a $25 \mathrm{~nm}$ structure within 3 pixels.

Recall that the ultimate goal of the MT tracking is not necessarily to track all MTs in a video, but to characterize a regulatory agent by quantifying the dynamic properties of a sufficiently large number of MTs. Therefore, algorithm evaluation is not a straightforward procedure in the biological relevance sense. For example, while the final performance depends upon the accuracy of the initial tip detection step, the tip detection may be designed to overestimate the number of candidates since the latter steps are constructed to eliminate inconsistencies among tip positions. In fact, we found that while the accuracy of the tracking algorithm

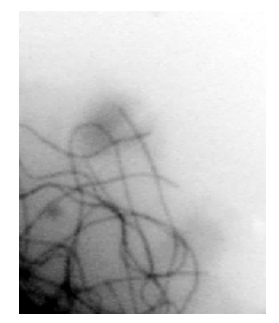

(a)

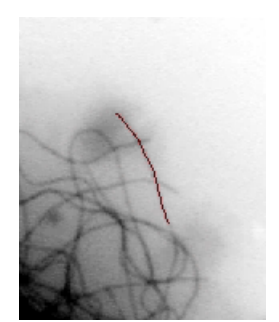

(d)

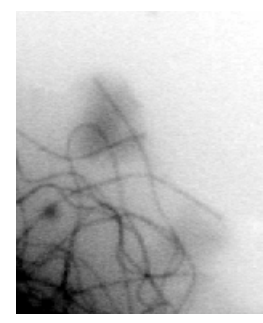

(b)

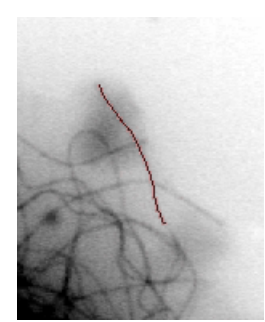

(e)

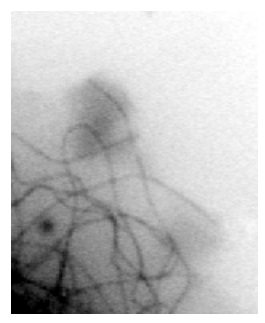

(c)

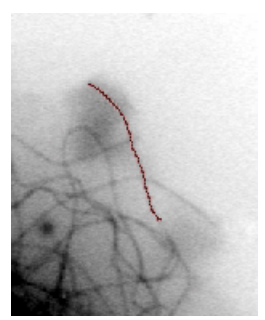

(f)
Figure 4. Example tracking results. Selected original frames are shown in the top row, with corresponding tracking results shown in the bottom row.

is not very high in matching the ground truth on individual MTs, the experimental conditions were found to be well separable and highly compatible with manual findings.

\subsection{User Assisted Tracking}

While the automated tracking delivers on increasing the number of tracked MTs, it attempts to extract the most trackable MTs regardless of their position. The central idea of the previous method rests on enhancing the polymer mass locations with a line filter for segmenting the MT body. This method operates on local intensity values for their conformance to a linear shape. Therefore, it is sensitive to frequent variations of intensity caused by additive fluorescence in crowded areas. The method discussed in this section, [10], addresses this issue by using an exploratory tracing algorithm which constraints a search space around the MT tip.

In essence, this approach formulates a deformable curve using the process trellis, [4], of a hidden Markov model (HMM). The transition matrix of the HMM encodes the lateral deformation potential of the curve and works much like active contours $[12,6]$. The emission probability distributions are used to match the current image content with the previous frame and the most probable path along the trellis is returned as the new trace of the MT body. Arcemission HMMs were found to perform better than regular (state-emission) HMMs for eliminating local jumps along the MT body. The initial curve is extracted by tracing the curve as detailed in [9]. The length changes are formulated with specialized HMM states, which determine to extend or stop the trace early. This algorithm consists of the following 
steps:

- tracing the MT body on initial frame,

- forming a spatial model of the trace,

- deforming the model of the previous frame based on current image observations.

This algorithm requires the $O$ and $T_{1}$ to extract the MT body in the first frame. Given the $O$ and $T_{1}$, the tracing algorithm explores an $(\alpha,-\alpha)$ neighborhood of $T_{1}$ in the opposite direction of the origin $O$, Fig. 5, and stops when explored pixels no longer satisfy the collected statistics along the curve. Essentially, the exploration consists of systematically examining the pixels within $(\alpha,-\alpha)$ of the line from $O$ to $T_{1}$ to determine the new direction for extension. While imposing a directional constraint the main contribution of working within the $(\alpha,-\alpha)$ neighborhood is to provide computational savings in exploration. In other words, it is possible to explore all directions starting form $T_{1}$ to capture sharp turns along the curve. But for MT bodies such turns are biophysically impossible.

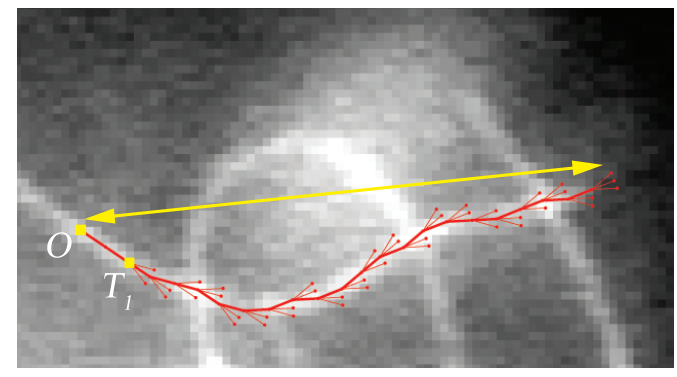

Figure 5. Example tracing from the origin $O$ towards $T_{1}$. Not all exploration paths are shown for clarity. The final MT length is computed along the curve rather than using the Euclidean distance.

Once the initial trace is complete a spatial model of the MT body is constructed in the first frame. This model is applied to the image content on the next frame to determine the deformations along the MT body, and the model is updated. The process is repeated over the frames of the video to compute the entire MT body track.

From a functional point of view, this method requires only the initial $O$ and $T_{1}$ to proceed with the tracking, while the previous method requires tip positions to be computed in all frames. While $O$ and $T_{1}$ could be estimated automatically, it is possible for the user to provide these points. This gives the user control over which MTs to track, adding to the biological utility of tracking.

\subsubsection{Evaluation of user assisted tracking}

Example tracking results are shown in Fig. 6. On 1374 traces, against the average of 4 human experts $\% 89$ of the MT tips were within 3 pixels of ground truth, with a mean error of 4.13 pixels.

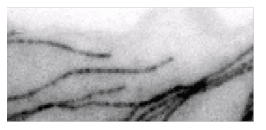

(a)

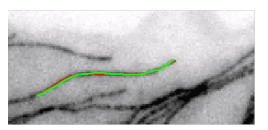

(d)

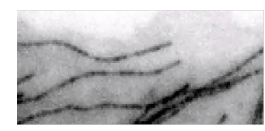

(b)

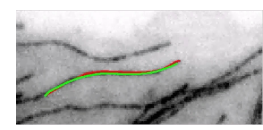

(e)

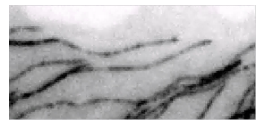

(c)

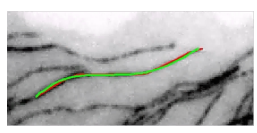

(f)
Figure 6. Example tracking results. Selected original frames are shown in the top row, with corresponding tracking results shown in the bottom row.

\section{Statistical evaluation of dynamic events}

In this section, we focus on evaluating dynamic characteristics from the tracking data. Upon the completion of the tracking procedure the MT lengths are plotted against time for each MT, Fig. 7. At this stage of the analysis, the operator segments the life history plot of each MT manually, deciding the boundaries for growth or shortening events. The rate of each segment of the life history plot is compared against two thresholds to be classified as a growth or shortening. Segments that do not meet the thresholds are classified as attenuation, signifying an uncertainty about the segment. Then, the segments of the same type are joined together for making up dynamic events, a consecutive stretch of segments. The rationale behind this procedure is to classify an event correctly within the context of the neighboring events in time. The final rate of an event is approximated between the beginning and the ending point of that event in time. Upon the completion of this segmentation, statistics are calculated for each event type. Typical statistics consist of average lengths and rates of growth and shortening as well as the frequencies of transitioning between the types of events. Finally, the dynamics characteristics of an agent is described using these statistics.

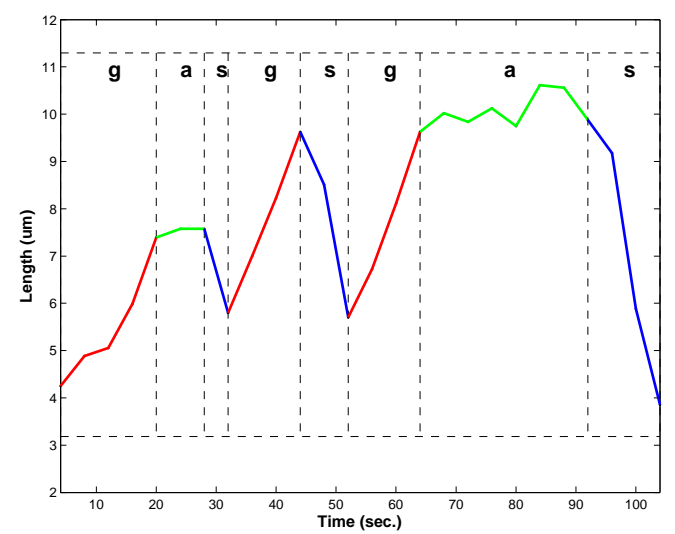

Figure 7. Life history plot of a MT. Dynamic events are labeled as (g) growth, (s) shortening, and (a) attenuation. 


\begin{tabular}{|l|c|}
\hline & Correct classification \\
\hline \hline Automated tracking & $95.91 \%$ \\
\hline Manual tracking & $92.16 \%$ \\
\hline
\end{tabular}

Table 1. Correct classification rates for automatically and manually extracted MT tracks.

Event boundaries can be estimated using dynamic programming. First, a cost function, such as the line-fitting error, is evaluated for all events, where an event is defined as any stretch of consecutive temporal segments starting at all time points. Then, a total cost of the segmentation is minimized for the entire life history. Rate or length thresholds can be applied to individual events for hard classification if necessary. Fig. 7 shows an example of this segmentation method. While this procedure is sufficient enough for automating the manual task, the collected statistics provide limited information about the temporal content. For example, it may be a hard problem to group similar event sequences.

As stated above, the analysis method does not consider events as part of a temporal pattern. As an example, consider the case of two hypothetical experimental conditions, each of which exhibit the same average growth and shortening rates. While these average rates may be equal, it is easy to realize that the underlying data may exhibit substantially discriminating information, possibly of high biological significance. For example, the transition frequencies from smaller rates to larger ones may be different between two experimental conditions, while scoring the same overall average rate for both. Using higher order statistical models may uncover such information.

Similar dynamics characteristics are known to be shared between different experimental conditions. Conversely, MTs within a cell exhibit different dynamic behavior patterns. This is the expected aggregate behavior since various MT groups perform different tasks. As an alternative analysis method, mixtures of HMMs were used in [3] for grouping time series based on their potential for representing a temporal pattern. Behavior patterns are established by clustering individual MT tracks into behavior categories. Mixtures of models represent the dynamic behaviors exhibited by an experimental condition. Individual MT tracks were assigned to an experimental condition based on their probabilities of being generated by the representative models.

This analysis method was tested on both automatically and manually extracted MT tracks for the ability to separate different experimental conditions. A total of 3068 automated tracks and 403 manual tracks were used. A maximum separation based on correct classification of a test set of MT tracks are shown in Table 1.

\section{Conclusion}

A straightforward approach to automate the MT dynamics analysis would be to replace the manual steps with their computational counterparts. To that end, a semi-automated procedure would present the researcher with a familiar environment and some degree of control. An alternative is to replace the entire analysis paradigm with computational methods. Here, we presented an example of each approach for the analysis of MT dynamic instability. The described tools are available at [1].

\section{References}

[1] http://www.bioimage.ucsb.edu. 5

[2] B. Alberts, A. Johnson, J. Lewis, M. Raff, K. Roberts, and P. Walter. Molecular Biology of the Cell. Garland Scientific, 4th edition, 2002. 1

[3] A. Altinok, M. El-Saban, A. J. Peck, L. Wilson, S. C. Feinstein, B. S. Manjunath, and K. Rose. Activity analysis in microtubule videos by mixture of hidden Markov models. CVPR, 2006 IEEE, 2:1662-1669, 2006. 2, 5

[4] Y. Chen, Y. Rui, and T. S. Huang. Multicue HMM-UKF for realtime contour tracking. IEEE TPAMI, 28(9):1525 - 1529, 2006. 3

[5] S. C. Feinstein and L. Wilson. Inability of tau to properly regulate neuronal microtubule dynamics: a loss-of-function mechanism by which tau might mediate neuronal cell death. Biochimica et Biophysica Acta - Molecular Basis of Disease, 1739(2-3):268-279, January 2005. 1

[6] C. Han, W. S. Kerwin, T. S. Hatsukami, J. Hwang, and C. Yuan. Detecting objects in image sequences using rulebased control in an active contour model. IEEE Transactions on Biomedical Engineering, 50(6):705-710, June 2003. 3

[7] S. Honore, K. Kamath, D. Braguer, S. B. Horwitz, L. Wilson, C. Briand, and M. A. Jordan. Synergistic suppression of microtubule dynamics by discodermolide and paclitaxel in non-small cell lung carcinoma cells. Cancer Research, (64):4957 - 4964, July 2004. 2

[8] K. Kamath, L. Wilson, F. Cabral, and M. A. Jordan. Biiitubulin induces paclitaxel resistance in association with reduced effects on microtubule dynamic instability. The Journal of Biological Chemistry, 280(13):12902-12907, April 2005. 2

[9] M. E. Sargin, A. Altinok, K. Rose, and B. S. Manjunath. Tracing curvilinear structures in live cell images. 6:285 288, Sep 2007. 3

[10] M. E. Sargin, A. Altinok, K. Rose, and B. S. Manjunath. Deformable trellis: open contour tracking in bioimage sequences. 2008. 2, 3

[11] J. Sethian. Level Set Methods and Fast Marching Methods. Cambridge Univ. Press, 1999. 3

[12] J. Tang and S. T. Acton. Vessel boundary tracking for intravital microscopy via multiscale gradient vector flow snakes. IEEE Transactions on Biomedical Engineering, 51(2):316324, February 2004. 3 